\title{
DETERMINANTES DE LA DIVERSIFICACIÓN EXPORTADORA: ENFOQUE BAYESIANO
}

\author{
Salinas Depaz, César ${ }^{A}$ | Pérez Cóndor, Wilder ${ }^{B}$ | Najarro Chuchón, Ricardo
}

\section{RESUMEN}

La diversificación exportadora brinda a las economías diversos beneficios como el fomento y estabilización del crecimiento económico mediante la reducción de la volatilidad de los ingresos por las exportaciones y el incremento de la productividad a través de externalidades positivas a nivel intra e inter sectorial. En este sentido, es relevante poder identificar qué posibilita la diversificación de las exportaciones; no obstante, ante un marco teórico disperso, esta identificación se convierte en una ardua tarea. Este documento mediante la metodología Bayesian Moving Average intenta descubrir qué factores idiosincráticos de cada país son relevantes para explicar una mayor diversificación exportadora en el largo plazo. Los resultados sugieren que seis elementos son los más importantes para la predicción de la diversificación de las exportaciones. Estos elementos son: i) renta de recursos naturales, ii) población, iii) formación bruta de capital (stock de capital), iv) enseñanza secundaria, v) crédito al sector privado y vi) capital humano. Estos resultados están en línea con los resultados obtenidos en la literatura.

JEL: O30, O47, G32.

PALABRAS CLAVES: Diversificación exportadora, Crecimiento Económico, Bayesian Moving Average Approach.

\begin{abstract}
Several benefits to the economy are provided by export diversification such as the promotion and stabilization of economic growth by reducing the volatility of export earnings and increasing productivity through positive externalities at the intra- and inter-sectorial levels. Therefore is important to identify what makes the diversification of exports possible. Thus, in this document, using the Bayesian Moving Average methodology tries to discover which idiosyncratic factors of each country are relevant to explain a greater export diversification in the long term. The results suggest that six elements are the most important to explain export diversification. These elements are: i) natural resources income, ii) population, iii) gross fixed capital formation (capital stock), iv) secondary education, v) financing to the private sector and vi) human capital. These results are in line with the results obtained in the literature.
\end{abstract}

JEL: O30, O47, G32.

KEYWORDS: Export Diversification, Economic Growth, Bayesian Moving Average Approach.

A César Salinas es Especialista en Investigación Económica en la Dirección de Política Macroeconómica y Descentralización Fiscal del Ministerio de Economia y Finanzas de Perú. Wilder Pérez es Especialista en Análisis Sectorial en la Dirección de Politica Macroeconómica y Descentralización Fiscal del Ministerio de Economía y Finanzas de Perú. Ricardo Najarro es Investigador Docente de la Escuela Profesional de Economia. Facultad de Ciencias Contables, Económicas y Financieras de la Universidad San Martín de Porres (USMP) y es Especialista en Proyecciones Macroeconómicas en la Dirección de Política Macroeconómica y Descentralización Fiscal del Ministerio de Economía y Finanzas de Perú.

B Los autores agradecen por sus valiosos comentarios a Wilder Ramirez Ventura y Carlos Montoro Llamosas. Los puntos de vista expresados en el presente trabajo de investigación corresponden a los autores y no reflejan necesariamente la posición de las instituciones en las que laboran. 


\section{INTRODUCCIÓN}

La literatura reciente en economía del desarrollo y comercio internacional destaca la importancia de la diversificación exportadora en la generación de efectos importantes en la productividad y el crecimiento económico. En efecto, una mayor diversificación de la canasta exportadora, en un contexto de apertura comercial, promueve el mayor crecimiento económico mediante el fomento de una mayor estabilidad en los ingresos por exportaciones, crea mayores encadenamientos entre sectores económicos, impulsa la creación de empleos de calidad y reduce la vulnerabilidad frente a choques de precios en mercados específicos ${ }^{12}$. Asimismo, la no concentración de exportaciones impulsa el crecimiento a través de externalidades positivas de la práctica o aprendizaje mediante la exportación (learning by exporting) y los potenciales efectos derrame ${ }^{3}$. Más aún, Lederman y Maloney (2003) encuentran que el efecto negativo de la abundancia de los recursos naturales en el crecimiento desaparece una vez que se controla por la concentración de las exportaciones.

Por tanto, debido a la importancia de la diversificación exportadora descrita en el párrafo anterior, el objetivo del presente documento de investigación es contribuir con evidencia empírica a la comprensión de los determinantes de la diversificación exportadora, para lo cual se tomó como información relevante el índice global de Theil calculado por el FMI, que mide el grado de concentración de exportaciones de una economía. Y dado que no existe un marco teórico único acerca de los principales determinantes de la diversificación, la incertidumbre en la especificación del modelo se determinan a través del enfoque bayesiano. En particular, se emplea la metodología de Bayesian Model Averaging (BMA, por sus siglas en inglés) para el periodo 1985-2010 y se considerarán 29 potenciales variables para 70 países $^{4}$.

1 Da Costa Neto, y R. Romeu (2011), "Did export diversification soften the impact of global financial crisis?", IMF Working paper, WP/11/99, Internacional Monetary Fund, Washington, DC.

2 Stanley, D y S. Bunnag (2001), "A new look at the benefits of diversification: lessons from Central America", Applied Economics, Taylor and Francis Journals, vol. 33 (11), pp 13691383.

3 Para el tema sobre vulnerabilidad, ver Barrot y Otros (2016), Jansen (2004; 2009), Jansen y Piermartini (2007). Con respecto al aprendizaje mediante la exportación y efectos derrame, ver Herzer y D. (2006), Hausmann et al. (2007) y Agosin $(2006,2009)$. Sobre la relación entre la diversificación de las exportaciones y el crecimiento, ver Al-Marhubi (2000), Lederman y Maloney (2003), Hesse (2008), o Cadot et al. (2012).

4 La elección de estas variables se hizo en base a estudios previos de Bebzuk y Berrettoni (2006), Ben Hammouda et al. (2006), Parteka (2010), Agosin et al. (2012), y Jetter y Ramirez
En este contexto, se vuelve relevante conocer las características de los determinantes más importantes de esta diversificación para la adecuada formulación de políticas de corto y largo plazo dirigidas al fomento de la competitividad mediante las ganancias de productividad que brinda una canasta exportadora más amplia a una economía.

El resto del documento está organizado de la siguiente manera. En la sección 2, revisamos brevemente el marco teórico asociado a temas de diversificación exportadora y crecimiento económico. La sección 3 describe en términos generales los datos utilizados en el presente trabajo de investigación, los cuales abarcan un periodo de análisis entre 1985-2010. La sección 4 proporciona una descripción de la metodología Bayesian Model Averaging (BMA, por sus siglas en inglés). Además, se describen los principales resultados obtenidos. Finalmente, se menciona algunas conclusiones en la sección 5 .

\section{MARCO TEÓRICO}

Existe una amplia literatura que relaciona a la diversificación exportadora $y$ el crecimiento económico; no obstante, el marco metodológico asociado con Bayesian Model Averaging es acotado. En este sentido, estudios como los de Agosin, M. (2009) y Cadot, O. et al. (2011) muestran que las economías con altos niveles de diversificación exportadora destacan por tener mejores tasas de crecimiento económico, elevados niveles de productividad y baja volatilidad de ciertas variables macroeconómicas. Estos resultados son significativos y robustos al utilizar diversos indicadores de diversificación exportadora.

Asimismo, Hesse, H. (2008), determinó el efecto positivo de la diversificación de las exportaciones sobre el crecimiento del ingreso per cápita. Este efecto es potencialmenteno lineal con los países en desarrollo que se benefician de la diversificación de sus exportaciones en contraste con los países avanzados que se benefician de la especialización en las exportaciones. Aditya, A. y Sinha Roy, S (2013) muestran que existe una relación entre la exportación y el crecimiento, teniendo en cuenta tanto la diversificación como la naturaleza de la composición de las exportaciones. Para ello, utilizan una muestra de 65 países para el período 1965-2005, la estimación del panel dinámico

(2015); así como la disponibilidad de estas variables para el periodo comprendido entre 1985 y 2010 . Estas variables están disponibles públicamente y provienen del Fondo Monetario Internacional, Banco Mundial, Penn World Table, Polity IV y NBER. 
revela que la diversificación y composición de las exportaciones son determinantes importantes del crecimiento económico después de controlar los impactos de otras variables como el rezago del crecimiento, las exportaciones, la inversión y la infraestructura. Lederman, D. y Maloney, F. (2003) encuentran que el efecto negativo de la abundancia de recursos naturales en el crecimiento desaparece una vez que controlan la concentración de las exportaciones.

Por su parte, Sala-i-Martin, J. (2004) examina la solidez de las variables explicativas en las regresiones de crecimiento económico entre países. Introduce y emplea un nuevo enfoque, Bayesian Averaging of Classical Estimates (BACE), que construye estimaciones promediando los coeficientes de OLS entre los modelos. Los pesos dados a las regresiones individuales tienen una justificación bayesiana similar al criterio de selección del modelo de Schwarz. En su documento de investigación, de las 67 variables explicativas, encuentra que
18 se correlacionan de manera significativa y robusta con el crecimiento a largo plazo y otras tres variables se relacionan marginalmente. La evidencia más sólida es el precio relativo de la inversión, la matriculación en la escuela primaria y el nivel inicial del PIB real per cápita.

Adicionalmente, diversos estudios empíricos proponen distintas variables que explican el comportamiento de la diversificación. Entre los principales determinantes de una mayor diversificación exportadora se destacan a la educación, población, crédito al sector privado, PBI y mayor gobernanza. Mientras que indicadores que se relacionan negativamente con la diversificación son la renta de recursos naturales, distancia a grandes mercados, apertura comercial, costos de exportación, transporte internacional y conflictos internos. Sin embargo, aún no se tiene consenso estricto respecto a cuáles son los principales determinantes de la diversificación exportadora.

Tabla 1

Determinantes de la diversificación exportadora

\begin{tabular}{|c|c|c|c|c|}
\hline Autor & $\begin{array}{c}\text { Variable } \\
\text { dependiente }\end{array}$ & $\begin{array}{l}\text { Nro. de } \\
\text { regresores }\end{array}$ & $\begin{array}{l}\text { Método } \\
\text { empírico }\end{array}$ & Determinantes relevantes \\
\hline $\begin{array}{l}\text { Jetter y } \\
\text { Ramírez } \\
(2015)\end{array}$ & HHI & 36 & BMA & $\begin{array}{l}\text { Educación primaria }(+) \text {, Recursos naturales }(-), \\
\text { Población }(+)\end{array}$ \\
\hline $\begin{array}{l}\text { Parteka y } \\
\text { Tamberi } \\
(2013)\end{array}$ & Gini, Theil & 34 & $\begin{array}{l}\text { OLS y IV- } \\
2 \text { SLS FE }\end{array}$ & $\begin{array}{c}\text { Población }(+) \text {, distancia a grandes mercados }(-) \text {, } \\
\text { PBI }(+) \text {, capacidad institucional en favor del } \\
\text { libre comercio }(+)\end{array}$ \\
\hline $\begin{array}{l}\text { Agosin, } \\
\text { Alvarez y } \\
\text { Bravo- } \\
\text { Ortega } \\
(2012)\end{array}$ & $\begin{array}{l}\text { HHI, Gini, } \\
\quad \text { Theil }\end{array}$ & 7 & $\begin{array}{c}\text { GMM } \\
\text { system } \\
\text { estimator }\end{array}$ & $\begin{array}{c}\text { Distancia económica }(-) \text {, apertura comercial }(-), \\
\text { escolaridad }(+) \text {, crédito al sector privado no } \\
\text { bancario }(+)\end{array}$ \\
\hline $\begin{array}{l}\text { Dennis y } \\
\text { Shepherd } \\
(2011)\end{array}$ & $\begin{array}{l}\text { Exportaciones } \\
\text { por sector }\end{array}$ & 23 & $\begin{array}{l}\text { Poisson, IV } \\
\text { Poisson y } \\
\text { 2SLS }\end{array}$ & $\begin{array}{l}\text { Costos de exportación }(-) \text {, entrada al mercado } \\
(-) \text {, transporte internacional }(-)\end{array}$ \\
\hline $\begin{array}{c}\text { Parteka } \\
(2010)\end{array}$ & $\begin{array}{l}\text { HHI, Gini, } \\
\text { Theil, } \\
\text { Coefficient of } \\
\text { variation }\end{array}$ & 2 & $\begin{array}{l}\text { Semiparamet } \\
\text { ric and } \\
\text { Nonparamet } \\
\text { ric methods }\end{array}$ & PBI (+), PBI squared (-) \\
\hline $\begin{array}{l}\text { Bebczuk y } \\
\text { Berrettoni } \\
(2006)\end{array}$ & HHI & 8 & OLS & $\begin{array}{l}\text { Productos primarios (-), GDP per cápita (+), } \\
\text { crédito al sector privado (+), América del Sur } \\
(-) \text {, África (-) }\end{array}$ \\
\hline $\begin{array}{l}\text { Hammouda, } \\
\text { Karingi, } \\
\text { Njuguna y } \\
\text { Jallab (2006) }\end{array}$ & HHI & 11 & OLS & $\begin{array}{l}\text { Formación bruta de capital (+), Form. Bruta de } \\
\text { capital squared (-), PBI }(+) \text {, PBI squared }(-), \\
\text { apertura comercial }(-) \text {, producción industrial }(+) \text {, } \\
\text { inflación }(-) \text {, tipo de cambio }(-) \text {, gobernanza }(+) \text {, } \\
\text { conflictos }(-)\end{array}$ \\
\hline
\end{tabular}

Fuente: Elaboración propia 
En línea con la literatura de crecimiento económico que utiliza el enfoque de BMA, en este estudio seleccionamos 30 variables utilizando dos criterios. Primero, nos quedamos con las variables que puedan representar a las variables de estado en un problema dinámico de optimización. Esto también nos permite lidiar, en parte, con alguna posible endogeneidad de los regresores. Por ello, elegimos variables medidas como el promedio de los cinco primeros años de nuestra muestra (1985-1989) con el propósito de corregir el problema de observaciones extremas. Esto hace que ciertas variables que no tienen observaciones para este periodo inicial no sean consideradas para los análisis de regresión efectuados más adelante.
El segundo criterio de selección utilizado se basa en un número igual de observaciones para todas las regresiones. Como varias variables no presentan información para distintos países, elegimos las 22 variables que maximizan el número de países con observaciones para todas estas variables.

Con estas restricciones, el tamaño final de la base de datos es de 23 variables (incluyendo a la variable dependiente definida como el promedio del índice de diversificación exportadora entre 1985 y 2010) para 70 países. Las variables, su descripción y la fuente de donde fueron extraídas se pueden ver en la tabla 2 .

Tabla 2

Descripción de la base de datos y fuentes

\begin{tabular}{|c|c|c|}
\hline Variable & Descripción y fuente & Media \\
\hline $\begin{array}{l}\text { Promedio del índice de } \\
\text { diversificación exportadora } \\
1985-2010\end{array}$ & $\begin{array}{l}\text { Índice global de Theil. Valores elevados asociados a una } \\
\text { menor diversificación. Fondo Monetario Internacional. }\end{array}$ & 3,54 \\
\hline Población & Población (en millones). Penn World Table. & 50,742 \\
\hline PBI real per cápita & $\begin{array}{l}\text { PBI real per cápita en poder de paridad de compra. Penn } \\
\text { World Table. }\end{array}$ & 6453,371 \\
\hline Tipo de cambio & Tipo de cambio, moneda nacional / dólar. Penn World Table. & 113,501 \\
\hline Tierra agrícola & Tierra agrícola (\% del área de tierra). Penn World Table. & 38,557 \\
\hline Tierra cultivable & Tierra cultivable (\% del área de tierra). Penn World Table. & 14,342 \\
\hline Enseñanza primaria & Matrícula escolar, primaria (\% bruto). Banco Mundial. & 91,422 \\
\hline Enseñanza secundaria & Matrícula escolar, secundaria (\% bruto). Banco Mundial. & 45,813 \\
\hline $\begin{array}{l}\text { Duración de educación } \\
\text { primaria }\end{array}$ & Educación primaria, duración (años). Banco Mundial. & 5,891 \\
\hline $\begin{array}{l}\text { Duración de educación } \\
\text { secundaria }\end{array}$ & Educación secundaria, duración (años). Banco Mundial. & 6,249 \\
\hline Nivel de democracia & $\begin{array}{l}\text { Nivel de democracia, }-10 \text { (totalmente autocrático) a }+10 \\
\text { (democracia total). Polity } I V .\end{array}$ & $-0,814$ \\
\hline Renta de recursos naturales & Renta total de recursos naturales (\% del PIB). Banco Mundial. & 6,691 \\
\hline Apertura comercial & $\begin{array}{l}\text { Exportaciones más importaciones (\% del PIB). Banco } \\
\text { Mundial. }\end{array}$ & 57,903 \\
\hline Crédito al sector privado & Crédito interno al sector privado (\% del PIB). Banco Mundial. & 33,541 \\
\hline Dinero amplio & Dinero amplio (\% del PIB). Banco Mundial. & 40,495 \\
\hline Cuenta corriente & Saldo de la cuenta corriente (\% del PIB). Banco Mundial. & $-3,353$ \\
\hline Formación bruta de capital & Formación bruta de capital (\% del PIB). Banco Mundial. & 20,633 \\
\hline Capital humano & Índice de capital humano. Penn World Table. & 1,9964 \\
\hline Inflación & Inflación, deflactor del PIB (\% anual). Banco Mundial. & 93,77 \\
\hline $\begin{array}{l}\text { Gasto de consumo del } \\
\text { gobierno general }\end{array}$ & $\begin{array}{l}\text { Gasto de consumo final del gobierno general (\% del PIB). } \\
\text { Banco Mundial. }\end{array}$ & 14,975 \\
\hline Inversión extranjera directa & $\begin{array}{l}\text { Inversión extranjera directa, entradas netas (\% del PIB). } \\
\text { Banco Mundial. }\end{array}$ & 0,671 \\
\hline Tipo de cambio fijo & $\begin{array}{l}\text { Clasificación estándar de Shambaugh } 2004 \text { ( } 1=\text { TC fijo, o = } \\
\text { no TC fijo). NBER dataset. }\end{array}$ & 0,354 \\
\hline
\end{tabular}

Fuente: Elaboración propia 
La tabla 3 contiene a todos los países considerados en la muestra. Además, incorpora el promedio del índice de Theil para el periodo 1985-2010 para estos países. Este indicador es una medida de la diversificación exportadora global de un país, tanto por el margen intensivo (within) como por el extensivo (between). Este cálculo lo realiza el
Fondo Monetario Internacional (FMI) siguiendo la definición y metodología descrita en Cadot, Carrère \& Strauss-Kahn (2011). Los países han sido ordenados de más a menos diversificados de acuerdo al promedio del índice de Theil para todo el periodo bajo análisis.

Tabla 3

Paises de la muestra con su indice de Theil promedio 1985-2010

\begin{tabular}{|c|c|c|c|c|c|}
\hline País & Theil & País & Theil & País & Theil \\
\hline Estados Unidos & 1.506 & Túnez & 3.099 & Gambia & 4.052 \\
\hline Reino Unido & 1.609 & Filipinas & 3.12 & Camerún & 4.15 \\
\hline Suiza & 1.849 & Egipto & 3.135 & Uganda & 4.299 \\
\hline China & 1.979 & Pakistán & 3.165 & Bangladesh & 4.354 \\
\hline Turquía & 2.012 & Nepal & 3.191 & Benín & 4.404 \\
\hline Canadá & 2.013 & Colombia & 3.194 & Venezuela & 4.489 \\
\hline Sudáfrica & 2.037 & Senegal & 3.194 & Comoros & 4.643 \\
\hline India & 2.037 & Costa Rica & 3.239 & Mauritania & 4.744 \\
\hline Tailandia & 2.095 & Bahrein & 3.243 & República Centroafricana & 4.766 \\
\hline Corea del Sur & 2.181 & Noruega & 3.259 & Malawi & 4.771 \\
\hline Argentina & 2.237 & Chile & 3.283 & Mali & 4.782 \\
\hline Uruguay & 2.332 & Fiyi & 3.331 & Guinea-Bissau & 4.788 \\
\hline México & 2.441 & Sri Lanka & 3.339 & Burundi & 4.951 \\
\hline Indonesia & 2.566 & El Salvador & 3.405 & Níger & 4.983 \\
\hline Jordán & 2.637 & Nicaragua & 3.413 & Argelia & 5.012 \\
\hline Israel & 2.726 & Honduras & 3.619 & Kuwait & 5.157 \\
\hline Malasia & 2.766 & Mauricio & 3.647 & Omán & 5.188 \\
\hline Guatemala & 2.852 & Trinidad y Tobago & 3.682 & Gabón & 5.406 \\
\hline Kenia & 2.859 & Madagascar & 3.684 & Irán & 5.412 \\
\hline Panamá & 2.975 & Togo & 3.745 & Chad & 5.425 \\
\hline Perú & 3.004 & Sierra Leona & 3.801 & Congo & 5.463 \\
\hline República Dominicana & 3.041 & Laos & 3.921 & Nigeria & 5.933 \\
\hline Zimbabue & 3.07 & Ghana & 3.99 & & \\
\hline Marruecos & 3.07 & Jamaica & 4.029 & & \\
\hline
\end{tabular}

Ordenados de más a menos diversificados.

Fuente: Elaboración propia

A partir de esta base de datos podemos confirmar la importancia de la diversificación exportadora en términos de distintas variables macroeconómicas. Parece existir una relación negativa entre crecimiento promedio del PBI per cápita y de la productividad total de factores con una mayor concentración de las exportaciones (ver gráficos 1 y 2), y una relación positiva entre la volatilidad promedio del crecimiento del producto y altos niveles de concentración en las exportaciones (ver gráficos 2). Por supuesto, esta simple observación no nos dice nada acerca de la relación causal entre estas variables. De hecho, la evidencia empírica al respecto muestra la existencia de una relación no lineal entre el producto y la diversificación de la producción (Imbs \& Wacziarg, 2003). Es decir, a medida que aumenta el PBI per cápita, la concentración de producción disminuye, pero después de alcanzar un cierto nivel de producto, la producción tiende a volverse más concentrada. A pesar de ello, es claro que una alta diversificación exportadora está asociada con mejores indicadores macroeconómicos, que finalmente consolida y mejora el entorno económico de un país. 
Gráfico 1.

Índice de concentración de exportaciones y crecimiento del PBI real per cápita (Promedio 1970-2010).

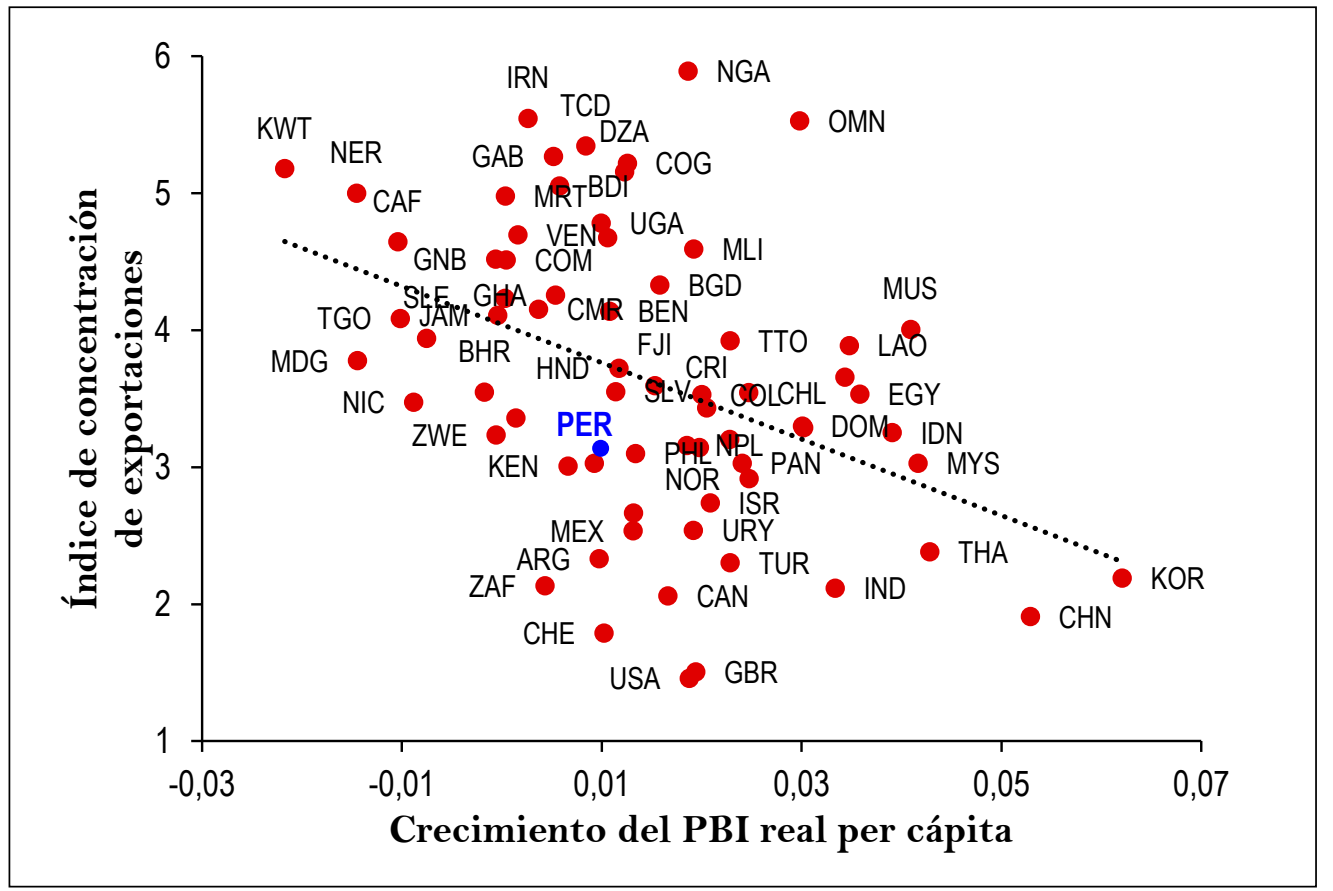

Fuente: Fondo Monetario Internacional y Penn World Table versión 9.0. Se utiliza el Índice de Theil para medir el grado de concentración en las exportaciones.

Gráfico 2.

Índice de concentración de exportaciones y crecimiento de la productividad total de factores (PTF) (Promedio 1970-2010).

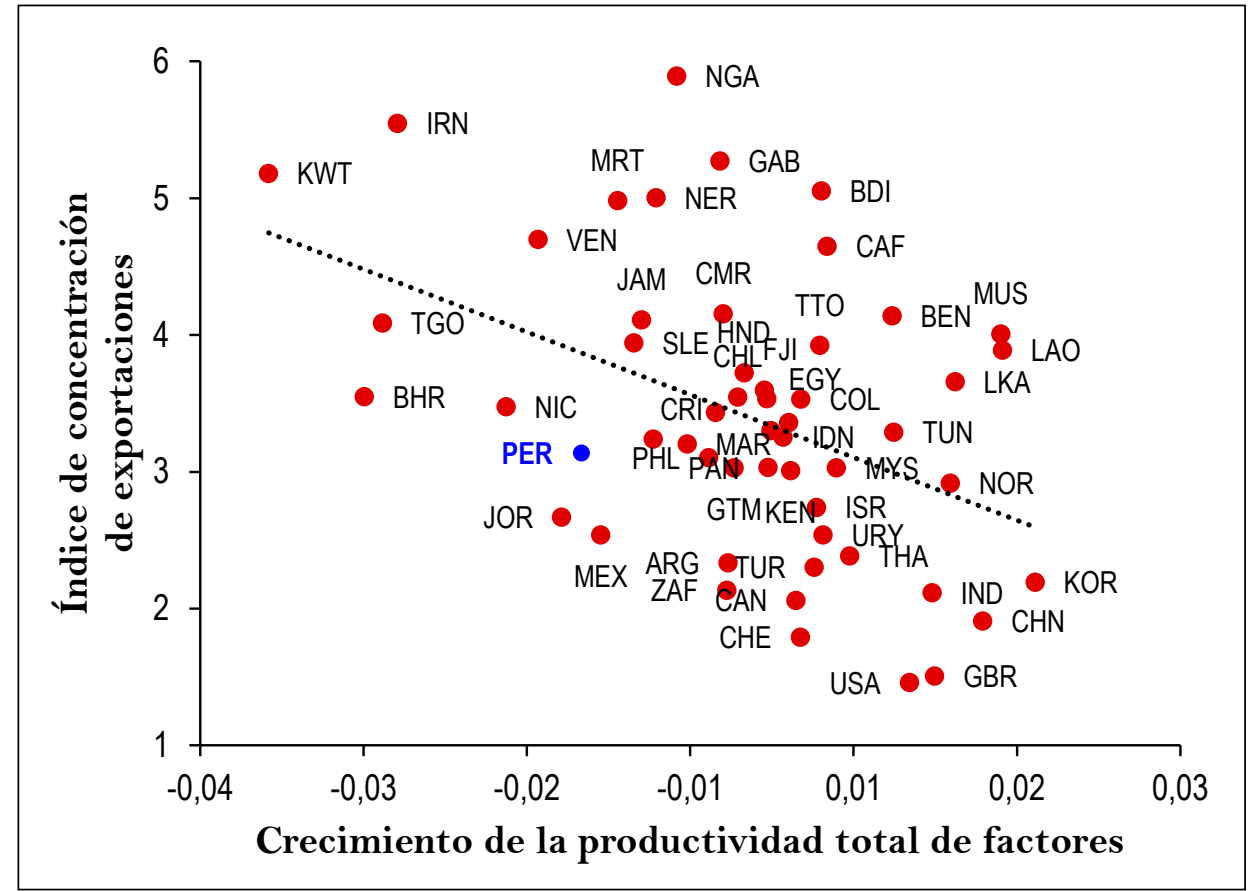

Fuente: Fondo Monetario Internacional y Penn World Table versión 9.0. Se utiliza el índice de Theil para medir el grado de concentración en las exportaciones. 
Gráfico 3. Índice de concentración de exportaciones y volatilidad del crecimiento del PBI real per cápita (Promedio 1970-2010).

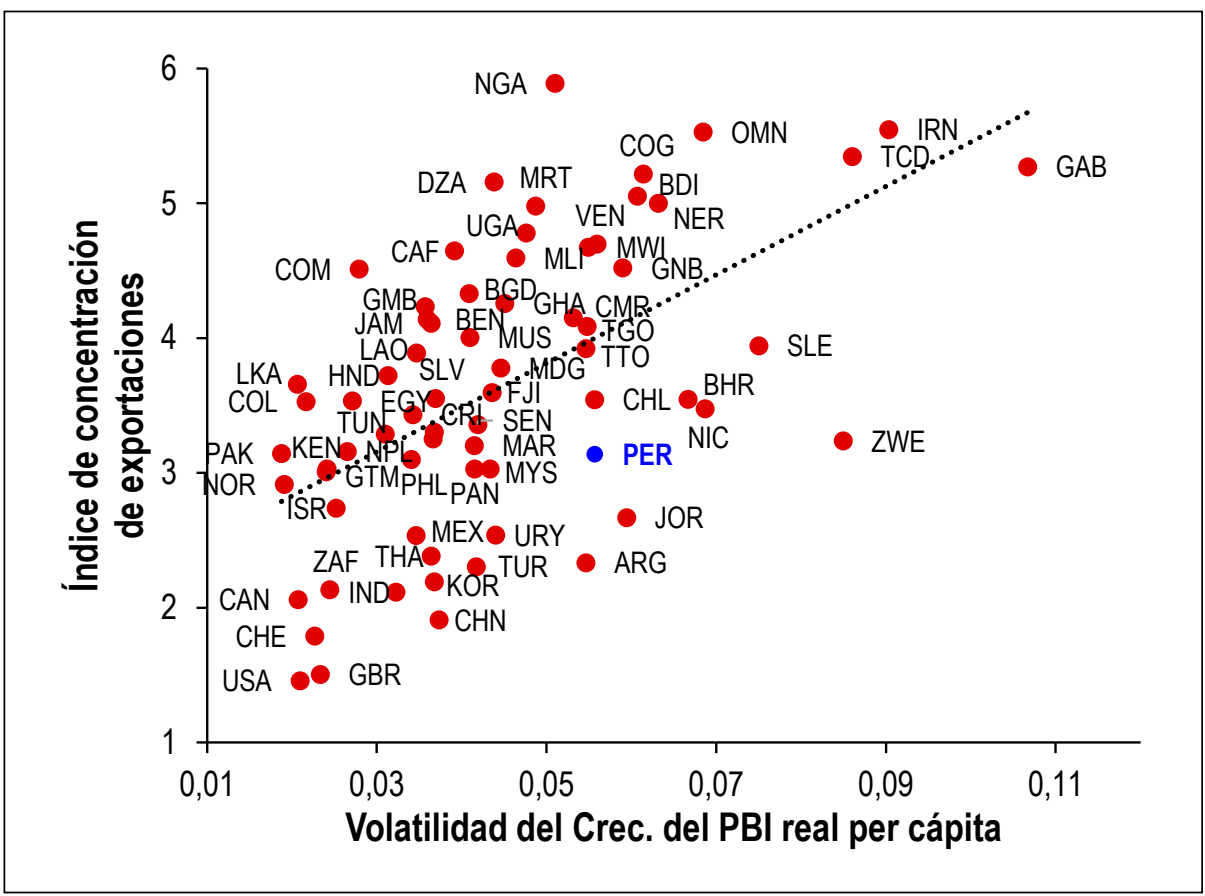

Fuente: Fondo Monetario Internacional y Penn World Table versión 9.0. Se utiliza el índice de Theil para medir el grado de concentración en las exportaciones.

\section{METODOLOGÍA ECONOMÉTRICA}

Siguiendo la literatura previa sobre exportación diversificada de Agosin, Alvarez \& BravoOrtega (2012) y Jetter \& Ramírez (2015), así como los estudios influyentes de Fernández, Ley \& Steel $(2001 ; 2001)$ y Sala-i-Martin, Doppelhofer \& Miller (2004), partiremos de un modelo de regresión lineal de la forma:

Ecuación 1:

$$
\boldsymbol{y}=\alpha \mathbf{1}_{n}+\boldsymbol{Z} \boldsymbol{\beta}+\boldsymbol{\varepsilon},
$$

Donde $\boldsymbol{y}$ es un vector que agrupa los índices de diversificación exportadora para $n$ países, $\boldsymbol{Z}=\left(\boldsymbol{z}_{1}, \boldsymbol{z}_{2}, \ldots, \boldsymbol{z}_{k}\right)$ es una matriz de variables explicativas de dimensión $n \times k, \boldsymbol{\beta}$ es un vector de coeficientes asociados a cada variable explicativa dentro de $\mathbf{Z}$, y $\boldsymbol{\varepsilon}$ es el vector de error que se supone está normalmente distribuido y es condicionalmente homocedástico $\varepsilon \mid \boldsymbol{X} \sim \mathbb{N}\left(\mathbf{0}, \sigma^{2} \boldsymbol{I}\right)$.

Dado que no existe un marco teórico único acerca de cuáles son específicamente los determinantes de la diversificación exportadora, existen $2^{k}$ modelos lineales posibles considerando que disponemos de $k$ potenciales regresores. Cada uno de estos modelos, denotados por $M_{j}$, depende de un vector de parámetros $\boldsymbol{\beta}_{j}$ que bajo el enfoque bayesiano están caracterizados por una función de distribución a priori $p\left(\boldsymbol{\beta}_{j} \mid M_{j}\right)$, una función de verosimilitud $p\left(\boldsymbol{y} \mid \boldsymbol{\beta}_{j}, M_{j}\right)$ y una función de distribución a posteriori $p\left(\boldsymbol{\beta}_{j} \mid \boldsymbol{y}, M_{j}\right)$.

En un contexto de incertidumbre en el modelo, es conocido que la elección de la función de distribución a priori puede tener un impacto sustancial sobre el resultado a posteriori. En este estudio seguiremos a Fernández, Ley \& Steel (2001) quienes, en base a resultados teóricos $\mathrm{y}$ simulaciones, proponen utilizar priors de referencia que tienen poca influencia sobre la inferencia y predicción a posteriori. En particular, estos autores proponen utilizar priors impropios no informativos para los parámetros comunes a todos los modelos $(\alpha, \sigma)$ y un $g$-prior para los parámetros $\boldsymbol{\beta}_{j}$. De esta forma, las distribuciones a priori son:

Modelo: $p\left(M_{j}\right)=1 / 2^{k} \propto 1$ (ningún modelo es favorecido a priori),

Varianza: $p\left(\sigma^{2}\right) \propto \sigma^{-2}$ (difuso, no informativo),

Intercepto: $p(\alpha) \propto 1$ (difuso, no informativo),

Pendientes: $\boldsymbol{\beta}_{j} \mid \boldsymbol{\alpha}, \sigma^{2} \propto \mathbb{N}\left(\mathbf{0}, \sigma^{2}\left(g_{j} \boldsymbol{Z}_{j}^{\prime} \boldsymbol{Z}_{j}\right)^{-1}\right)(g$-prior). 
Respecto a la calibración de $g_{j}$, se puede notar que si $g_{j}=0$ el prior de $\boldsymbol{\beta}_{j}$ es completamente difuso. Por el contrario, si $g_{j}=1$ se le asigna la misma importancia al prior que a la información muestral. Fernández, Ley \& Steel (2001) discuten varias posibilidades y recomiendan utilizar $g_{j}=1 / \operatorname{máx}\left\{n, k^{2}\right\}$. Note que en la especificación de la varianza y el intercepto asumiremos un prior común para todos los diferentes modelos. Esto se debe a que los componentes de $\boldsymbol{\beta}$ que no aparecen en $M_{j}$ se asumen que son iguales a cero y por tanto, siempre estamos condicionando los parámetros sobre el conjunto completo de regresores $\boldsymbol{Z}$, lo cual otorga la misma interpretación de $\sigma$ y $\alpha$ en todos los posibles modelos.

Un aspecto fundamental de esta aplicación es que nos enfrentamos ante la incertidumbre en la elección de los regresores. Esto significa que también necesitamos especificar una distribución a priori de cada uno de los posibles modelos. En la especificación del modelo líneas arriba, el espacio de modelos se distribuye de manera uniforme en donde la probabilidad de ocurrencia de cada modelo es igual a $1 / 2^{k}$.

La elección de estos priors permite obtener resultados analíticos y reducir considerablemente la carga computacional. Definiendo $\boldsymbol{X}_{j}=\left(\boldsymbol{Z}_{j} \mathbf{1}_{n}\right)$, $N_{j}=I_{n}-X_{j}\left(X_{j}^{\prime} X_{j}\right)^{-1} X_{j}^{\prime}$ y $N_{1}=I_{n}-\mathbf{1}_{n} 1_{n}^{\prime} / n$, los resultados $a$ posteriori son:

Ecuación 2:

$$
\mathbb{E}\left(\boldsymbol{\beta}_{j} \mid \boldsymbol{y}, M_{j}\right)=\frac{1}{1+g_{j}}\left(\boldsymbol{Z}_{j}^{\prime} \boldsymbol{Z}_{j}\right)^{-1} \boldsymbol{Z}_{j}^{\prime} \boldsymbol{y},
$$

Ecuación 3:

$$
\mathbb{V}\left(\boldsymbol{\beta}_{j} \mid y, M_{j}\right)=\frac{1}{1+g_{j}}\left(\frac{S_{j}^{*}}{n-2}\right)\left(\boldsymbol{Z}_{j}^{\prime} \boldsymbol{Z}_{j}\right)^{-1},
$$

Ecuación 4:

$$
S_{j}^{*}=\left(\frac{1}{1+g_{j}}\right) \boldsymbol{y}^{\prime} N_{j} \boldsymbol{y}+\left(\frac{g_{j}}{1+g_{j}}\right) \boldsymbol{y}^{\prime} N_{1} \boldsymbol{y},
$$

Ecuación 5:

$$
p\left(y \mid M_{j}\right) \propto\left(\frac{g_{j}}{1+g_{j}}\right)^{\frac{k_{j}}{2}}\left(S_{j}^{*}\right)^{-\frac{n-1}{2}},
$$

Esta última distribución a posteriori también puede ser calculada utilizando una calibración Bayesiana empírica. Este principio es similar a una estimación por máxima verosimilitud: se elige el valor del hiperparámetro en cuestión que maximiza la función de verosimilitud marginal:

Ecuación 6:

$\ln p\left(\boldsymbol{y} \mid M_{j}\right)=C+\frac{k_{j}}{2} \ln \left(\frac{g_{j}}{1+g_{j}}\right)-\frac{n-1}{2} \ln \left(\boldsymbol{y}^{\prime} N_{j} y+\frac{g_{j}}{1+g_{j}} \boldsymbol{y}^{\prime}\left(N_{1}-N_{j}\right) y\right)$.

El enfoque bayesiano automáticamente lidia con la incertidumbre del modelo. Sea $\boldsymbol{\phi}$ un vector de parámetros con la misma interpretación en todos los modelos. Cada modelo generará una distribución a posteriori de la forma $p\left(\boldsymbol{\phi} \mid \boldsymbol{y}, M_{j}\right)$. Tomando en cuenta las leyes de probabilidad usuales, la inferencia multimodelo $\boldsymbol{\phi}$ de se basa en la siguiente distribución a posteriori:

Ecuación 7:

$$
p(\boldsymbol{\phi} \mid \boldsymbol{y})=\sum_{j=1}^{2^{k}} p\left(\boldsymbol{\phi} \mid \boldsymbol{y}, M_{j}\right) p\left(M_{j} \mid \boldsymbol{y}\right),
$$

Donde $p\left(M_{j} \mid \boldsymbol{y}\right)$ es la probabilidad a posteriori para el modelo $j$. Más aún, si $f(\boldsymbol{\phi})$ es una función de, las reglas $\boldsymbol{\phi}$ de expectativas condicionales indican que:

Ecuación 8:

$$
\mathbb{E}(f(\boldsymbol{\phi}) \mid \boldsymbol{y})=\sum_{j=1}^{2^{k}} \mathbb{E}\left(f(\boldsymbol{\phi}) \mid \boldsymbol{y}, M_{j}\right) p\left(M_{j} \mid \boldsymbol{y}\right),
$$

Con ello es posible calcular medias, percentiles y otros estadísticos a posteriori no condicionales a un modelo en específico. Del Teorema de Bayes, se tiene que la probabilidad a posteriori del modelo $j$ es:

Ecuación 9:

$$
p\left(M_{j} \mid \boldsymbol{y}\right)=C p\left(\boldsymbol{y} \mid M_{j}\right) p\left(M_{j}\right),
$$

Donde $p\left(M_{j}\right)$ es la probabilidad a priori del modelo $j, p\left(\boldsymbol{y} \mid M_{j}\right)$ es la función de verosimilitud marginal del modelo y $C$ es una constante de integración (no depende de $j$ y es común para todos los modelos). Como el espacio de modelo es discreto, se puede determinar $C$ ya que $\sum_{j=1}^{2^{k}} p\left(M_{j} \mid y\right)=1$ y la probabilidad a posteriori del modelo $j$ es:

Ecuación 10:

$$
p\left(M_{j} \mid \boldsymbol{y}\right)=\frac{p\left(\boldsymbol{y} \mid M_{j}\right) p\left(M_{j}\right)}{\sum_{i=1}^{S} p\left(M_{i} \mid \boldsymbol{y}\right) p\left(M_{i}\right)^{\prime}}
$$

A pesar de que se disponen de expresiones como esta, la probabilidad a posteriori puede resultar computacionalmente inmanejable. 
Por ejemplo, en nuestra aplicación, $k=29$ potenciales regresores y necesitaríamos calcular la probabilidad a posteriori para cada uno de $\operatorname{los} 2^{29}=536870912$ modelos y promediar las distribuciones requeridas sobre todos estos modelos. En orden de reducir sustancialmente la carga computacional, aproximaremos la distribución a posteriori simulando una muestra de todos los potenciales modelos aplicando la metodología de Markov Chain Monte Carlo Model Composition $\left(\mathrm{MC}^{3}\right)$ propuesta por Madigan \& York (1995). El propósito del algoritmo $\mathrm{MC}^{3}$ es evitar evaluar todos los $2^{k}$ modelos, tomando muestras del espacio de modelos. Si se hace esto un gran número de veces ( $S$ veces, $S<2^{k}$ ) se puede aproximar $p\left(M_{j} \mid \boldsymbol{y}\right)$ con:

Ecuación 11:

$$
p\left(M_{j} \mid \boldsymbol{y}\right)=\frac{p\left(\boldsymbol{y} \mid M_{j}\right) p\left(M_{j}\right)}{\sum_{i=1}^{S} p\left(M_{i} \mid \boldsymbol{y}\right) p\left(M_{i}\right)^{\prime}}
$$

El algoritmo de $\mathrm{MC}^{3}$ se basa en el muestreo de Metropolis-Hastings, formando una cadena de Markov sobre el conjunto total de posibles modelos. Esta cadena de Markov está diseñada para que los modelos que visite sean los que tienen una mayor probabilidad a posteriori. Así, con $S$ suficientemente grande, se tiene que los modelos no visitados por la cadena son, comparativamente, irrelevantes.

\section{RESULTADOS}

Siguiendo la sección de metodología de este documento, aplicaremos el enfoque BMA utilizando el algoritmo de $\mathrm{MC}^{3}$. El número de repeticiones burn-in son 60000 y el número de repeticiones a ser utilizadas en el muestreo es de 30 0000. El algoritmo visitó 15026 modelos únicos de $2^{29}$ modelos potenciales y la correlación entre la probabilidad a posteriori de los 20 mejores modelos calculadas analítica y numéricamente es de 0,996 (ver gráfico 4), indicando el buen desempeño del algoritmo (Fernández, Ley, \& Steel, 2001).

La tabla 4 muestra los resultados a posteriori del modelo de BMA. A partir de estos resultados es claro que las seis principales predictores de la exportación diversificada son la renta de recursos naturales, población, formación bruta de capital, enseñanza secundaria, crédito al sector privado, y capital humano. La probabilidad de inclusión a posteriori de las primeras tres variables es de $1,00,0,925$ y 0,916 , respectivamente, lo cual significa que estas variables están incluidas en el $100 \%, 92,5 \%$ y $91,6 \%$ de las veces en los mejores modelos, respectivamente. Luego esta probabilidad cae hasta por encima de 0,5 para las tres variables restantes.

Sin embargo, dado que tenemos las distribuciones de probabilidad de cada una de estas variables, es posible calcular ratios $t$ que puedan dar cuenta de la importancia relativa de estas mismas. Comparando estos estadísticos con el valor crítico de la prueba $\mathrm{t}$ a dos colas $(1,96)$, es notoria la relevancia de estas seis variables en el espacio de los mejores modelos propuestos. El gráfico 5 muestra la distribución a posteriori del tamaño del modelo y confirma que la diversificación exportadora está determinada principalmente por estas seis variables.

Gráfico 4. Probabilidad a posteriori de los 20 mejores modelos $(\rho=0,99775)$.

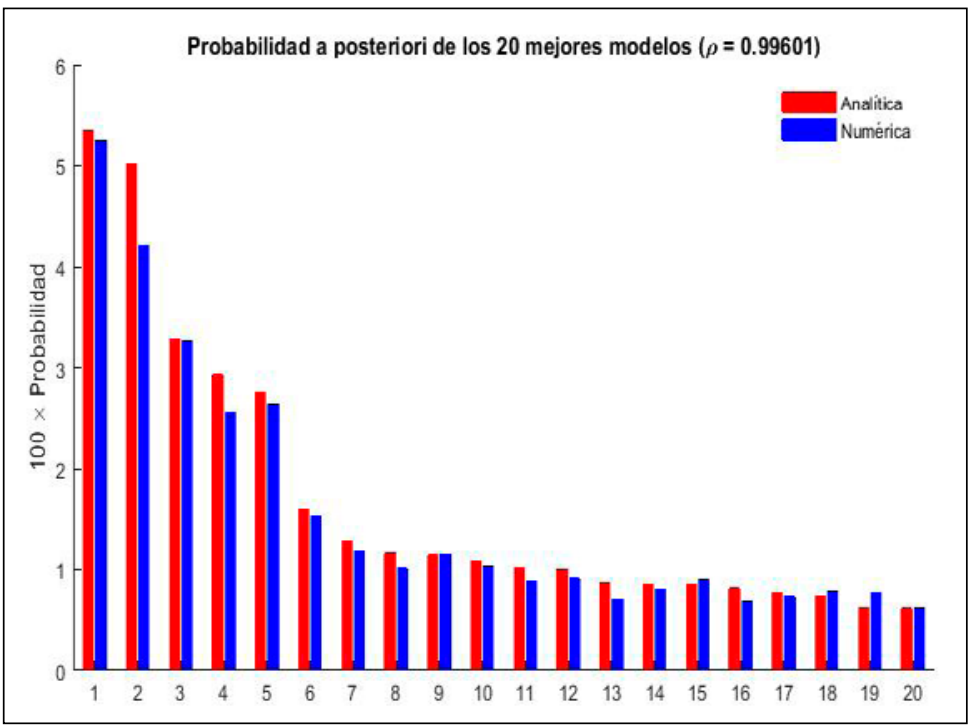

Fuente: Fondo Monetario Internacional y Penn World Table versión 9.0. Se utiliza el índice de Theil para medir el grado de concentración en las exportaciones. 
Gráfico 5. Distribución del tamaño del modelo.

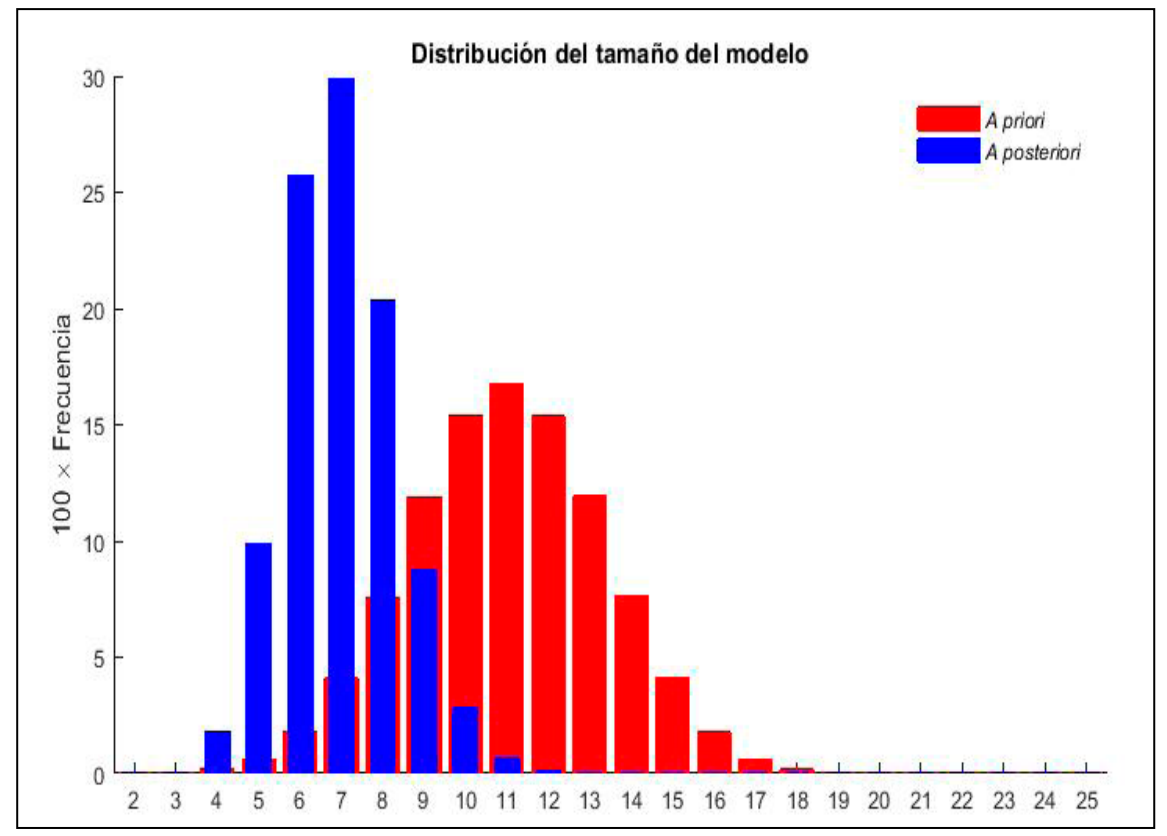

Fuente: Fondo Monetario Internacional y Penn World Table versión 9.0. Se utiliza el índice de Theil para medir el grado de concentración en las exportaciones.

Para dar cuenta de las relaciones de estos determinantes con la variable explicada, la columna 3 de la tabla 4 muestra la media a posteriori promedio de la distribución de los efectos de cada una de estas variables sobre el indicador de diversificación exportadora. Considerando el signo de esta medida, altos niveles en la renta de recursos naturales están asociados con una menor diversificación exportadora ${ }^{5}$. Por el contrario, los países con mayor población (mayor oferta laboral) tienen, en general, una canasta exportadora menor concentrada que el resto de países. Por su parte, los países con una alta formación bruta de capital (stock de capital) se asocian con una mayor especialización en sus canastas exportadoras. No obstante, una mayor matrícula escolar a nivel secundaria, un alto grado de profundización financiera (crédito al sector privado) y un alto desarrollo del capital humano incrementan la diversificación exportadora local ${ }^{6}$.

\footnotetext{
5 Un mayor nivel implica una mayor (menor) concentración (diversificación) exportadora.

6 Todas las variables explicativas (regresores) se pueden considerar como variables predeterminadas (exógenas) ya que solamente se han considerado los valores de estas variables para el periodo 1985-1989, mientras que la explicativa es el promedio del indicador para todo el periodo bajo análisis (1985-2010).
}

Tabla 4

Resultados a posteriori (promedio de modelos)

\begin{tabular}{lccc}
\hline Variable & Prob.de inclusión a posteriori & Media & Ratio t \\
\hline Renta de recursos naturales & 1.000 & 7.5508 & 7.1539 \\
Población & 0.925 & -0.1466 & 3.4755 \\
Formación bruta de capital & 0.916 & 26.6980 & 3.3978 \\
Enseñanza secundaria & 0.553 & -0.9433 & 3.1313 \\
Crédito al sector privado & 0.496 & -0.5375 & 2.0317 \\
Capital humano & 0.493 & -38.6305 & 3.0279 \\
Dinero amplio & 0.262 & -0.2278 & 1.2490 \\
Duración de la secundaria & 0.188 & 3.4700 & 0.8311 \\
Gasto de consumo del gobierno & 0.141 & 0.3330 & 0.6748 \\
Intervención cambiaria & 0.109 & 2.9447 & 0.5056 \\
Tierra agrícola & 0.107 & -0.0620 & 0.5024 \\
Inflación & 0.101 & -0.0620 & 0.5034 \\
Grado de democracia & 0.096 & -0.1847 & 0.4512 \\
Duración de la primaria & 0.074 & -1.2231 & 0.3279 \\
Enseñanza secundaria & 0.070 & -0.0347 & 0.3449 \\
Apertura comercial & 0.055 & 0.0122 & 0.2020 \\
PBI per cápita & 0.052 & 0.0000 & 0.0160 \\
Tierra cultivable & 0.050 & 0.0214 & 0.1615 \\
Tipo de cambio & 0.045 & -0.0009 & 0.1301 \\
Cuenta corriente & 0.045 & -0.0055 & 0.0220 \\
Inversión extranjera directa & 0.041 & -0.1250 & 0.0964 \\
\hline
\end{tabular}

Fuente: Elaboración propia

Desagregando estos resultados para el caso de los tres mejores modelos, elegidos en términos de su probabilidad a posteriori, podemos concluir que los resultados del enfoque BMA son consistentes para estos modelos (ver tabla 4). El mejor modelo incluye a cinco de las seis variables más importantes discutidas anteriormente: renta de recursos naturales, población, formación bruta de capital, enseñanza secundaria y créditos al 
sector privado. El segundo mejor modelo excluye una variable del primer modelo y la reemplaza por el capital humano. El tercer mejor modelo únicamente excluye al variable crédito al sector privado.

Tabla 5

Resultados a posteriori (mejores 3 modelos)

\begin{tabular}{lccccc}
\hline \multicolumn{1}{c}{ Variable } & $\begin{array}{c}\text { Prob.de inclusión a } \\
\text { posteriori }\end{array}$ & Media & Ratio t & BMA m & BMA t \\
\hline & Mejor modelo 1 & & & & \\
\hline Recursos naturales & 1.000 & 8.3894 & 8.4194 & 7.5505 & 7.1538 \\
Población & 0.925 & -0.1508 & 3.4609 & -0.1466 & 3.4756 \\
Formación bruta de capital & 0.916 & 31.1736 & 3.8193 & 26.7124 & 3.3986 \\
Enseñanza secundaria & 0.553 & -1.7181 & 4.7063 & -38.6157 & 3.0251 \\
Crédito al sector privado & 0.497 & -0.9866 & 2.7303 & -0.2273 & 1.2472 \\
\hline & Mejor modelo 2 & & & \\
\hline Recursos naturales & 1.000 & 7.033 & 6.7926 & 7.5505 & 7.1538 \\
Población & 0.925 & -0.1577 & 3.7203 & -0.1466 & 3.4756 \\
Formación bruta de capital & 0.916 & 30.5094 & 3.7371 & 26.7124 & 3.3986 \\
Capital humano & 0.493 & -88.5519 & 6.2073 & -38.6157 & 3.0251 \\
Enseñanza secundaria & 0.262 & -0.9032 & 2.7498 & -0.2273 & 1.2472 \\
\hline & Mejor modelo 3 & & & & \\
\hline Recursos naturales & 1.000 & 8.2623 & 7.8560 & 7.5505 & 7.1538 \\
Población & 0.925 & -0.1831 & 4.1320 & -0.1466 & 3.4756 \\
Formación bruta de capital & 0.916 & 27.2525 & 3.2100 & 26.7124 & 3.3986 \\
Enseñanza secundaria & 0.553 & -2.3220 & 7.5671 & -0.9433 & 3.1307 \\
\hline
\end{tabular}

Fuente: Elaboración propia

\section{CONCLUSIONES}

En el presente trabajo de investigación, en una primera instancia, mostramos a través de un análisis de correlaciones simples la importancia de la diversificación exportadora en distintas variables macroeconómicas como el crecimiento económico y la productividad total de factores.

Asimismo, a través del uso del marco metodológico asociado con Bayesian Model Averaging, encontramos que, de un total de 22 variables, cinco variables son las más relevantes para impulsar políticas de diversificación exportadora en un país. Dichas variables se enumeran a continuación: i) renta de recurso naturales; ii) población, la cual está asociada a la mayor oferta laboral; iii) formación bruta de capital, que se traduce en un incremento del stock de capital físico, que permitiría una mayor especialización en las canastas exportadoras de los países; iv) enseñanza secundaria, medido a través de un mayor nivel educativo; v) crédito al sector privado, asociado a un alto grado de profundización financiera; y vi) capital humano.

En este contexto, los determinantes de la diversificación exportadora se vuelven relevantes para la adecuada formulación de políticas económicas de corto, mediano y largo plazo dirigidas al fomento de la competitividad mediante las ganancias de productividad que brinda una canasta exportadora más amplia a una economía.

\section{REFERENCIAS}

[1] Abiad, A., Furceri, D., y Topalova, P. (2015). The macroeconomic effects of public investment: evidence from advanced economies. IMF Working Paper, 15(95).

[2] Abrigo, M. R., y Love, I. (2016), Estimation of panel vector autoregression in Stata: a Package of Programs. The Stata Journal, 16(3), pp. 778-804.

[3] Aditya, A. y Sinha Roy, S. (2013), Export Diversification and Economic Growth: Evidence from Cross-Country Analysis. The Journal of International Trade \& Economic Development, 22(7). doi: 10.1080/09638199.2011.619009

[4] Agosin, M. (Abril de 2009). Export diversification and growth in emerging economies. CEPALReview, (97), pp. 115-131. 
[5] Agosin, M. R., Alvarez, R., y BravoOrtega, C. (2012). Determinants of export diversification around the world: 1962-2000. The World Economy, 35(3), pp. 295-315. doi: $10.1111 / \mathrm{j} .1467-9701.2011 .01395 . \mathrm{x}$

[6] Anderson, T. W., y Hsiao, C. (1982). Formulation and estimation of dynamic models using panel data. Journal of Econometrics, 18(1), pp. 47-82.

[7] Andrews, D. W., y Lu, B. (2001). Consistent model and moment selection procedures for GMM estimation with application to dynamic panel data models. Journal of Econometrics, 101(1), pp. 123-164.

[8] Arellano, M., y Bover, O. (1995). Another look at the instrumental variable estimation of error-components models. Journal of Econometrics, 68(1), pp. 29-51.

[9] Bebczuk, R. N., y Berrettoni, D. (2006). Explaining export diversification: an empirical analysis. Departamento de Economía, Facultad de Ciencias Económicas, Universidad Nacional de La Plata, (65).

[10] Ben Hammouda, H., Karingi, S., Njuguna, A., y Sadni Jallab, M. (2006). Diversification: towards a new paradigm for Africa's development. African Trade Policy Centre, Economic Commission for Africa, (35).

[11] Blundell, R., y Bond, S. (1998). Initial conditions and moment restrictions in dynamic panel data models. Journal of Econometrics, 87(1), pp. 115-143.

[12] Cadot, O., Carrère, C., y Strauss-Kahn, V. (2011). Export diversification: what's behind the hump? The Review of Economics and Statistics, 93(2), pp. 590-605.

[13] Canova, F., y Ciccarelli, M. (2009). Estimating multicountry VAR models. International Economic Review, 50(3), pp. 929-959.

[14] Dennis, A., y Shepherd, B. (2011). Trade facilitation and export diversification. The World Economy, 34(1), pp. 101-122.

[15] Fernández, C., Ley, E., y Steel, M. F. (2001). Benchmark priors for Bayesian model averaging. Journal of Econometrics, 100(2), pp. 381-427.
[16] Fornero, J., Kirchner, M., y Yany, A. (2016). Terms of Trade Shocks and Investment in Commodity-Exporting Economies. Central Bank of Chile, (773).

[17] Gondo, R., y Vega, M. (2017). The dynamics of investment projects: evidence from Peru. Bank for International Settlements, (621). Banco Central de Reserva del Perú, (6).

[18] Holtz-Eakin, D., Newey, W., y Rosen, H. S. (1988). Estimating vector autoregressions with panel data. Econometrica, 56(6), pp. 1371-1395.

[19] Hesse, H. (2008). Export Diversifi cation and Economic Growth. The World Bank, (21).

[20] Imbs, J., y Wacziarg, R. (2003). Stages of diversification. American Economic Review, 93(1), pp. 63-86.

[21] Jetter, M., y Ramírez Hassan, A. (2015). Want export diversification? Educate the kids first. Economic Inquiry, 53(4), pp. 1765-1782.

[22] Judson, R. A., y Owen, A. L. (1999). Estimating dynamic panel data models: A guide for macroeconomists. Economics Letters, 65(1), pp. 9-15.

[23] Lederman, D. y Maloney, W. (2007). Trade Structure and Growth. World Bank, (3025).

[24] Lütkepohl, H. (2005). New Introduction to Multiple Time Series. Berlin, Alemania: Springer.

[25] Madigan, D., y York, J. (1995). Bayesian graphical models for discrete data. International Statistical Review, 63(2), pp. 215-232.

[26] Nickell, S. (1981). Biases in dynamic models with fixed effects. Econometrica, 49(6), pp. 1417-1426.

[27] Ozan Yildirim, M., y Eren Yildirim, A. (2017). The influence of consumption and investment on unemployment in Turkey: a SVAR approach. EKONOMIKA, 96(1), pp. 74-92. doi: https://doi.org/10.15388/ ekon.2017.1.10665 
[28] Parteka, A. (2010). Employment and export specialisation along the development path: some robust evidence. Review of World Economics, 145(4), pp. 615-640. doi: $10.1007 / \mathrm{s} 10290-009-0037-y$

[29] Parteka, A., y Tamberi, M. (2013). What determines export diversification in the development process? Empirical assessment. The World Economy, 36(6), pp. 807-826.
[30] Sala-i-Martin, X., Doppelhofer, G., y Miller, R. I. (2004). Determinants of long-term growth: a Bayesian Averaging of Classical Estimates (BACE) approach. The American Economic Review, 94(4), pp. 813-835.

[31] Sims, C. A. (1980). Macroeconomics and reality. Econometrica, 48(1), pp. 1-48. doi: $10.2307 / 1912017$ 